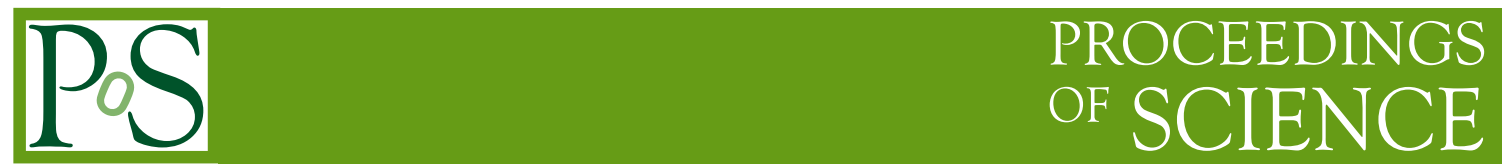

\title{
Perturbative contributions to rare B-meson decays
}

\author{
Mikolaj Misiak*i \\ University of Warsaw \\ E-mail: misiakefuw.edu.pl
}

Loop-mediated rare $B$-meson decays provide sensitive tests of the Standard Model and constraints on its extensions. In this contribution, I present a brief review of several recent perturbative calculations that contribute to precise theoretical predictions for $B_{s, d} \rightarrow \ell^{+} \ell^{-}, B \rightarrow X_{s} \gamma$ and $B \rightarrow$ $X_{s} \ell^{+} \ell^{-}$.

XXII. International Workshop on Deep-Inelastic Scattering and Related Subjects 28 April - 2 May 2014

Warsaw, Poland

*Speaker.

$\dagger$ This work was supported in part by the National Science Centre (Poland) research project, Decision No. DEC2011/01/B/ST2/00438. The $B_{s, d} \rightarrow \ell^{+} \ell^{-}$part is based on the article [1] prepared together with C. Bobeth, M. Gorbahn, T. Hermann, E. Stamou and M. Steinhauser. 
Rare $B$-meson decays are well known to provide sensitive tests of the Standard Model (SM) and important constraints on its extensions. Since they occur at scales $\mu \ll M_{W}$, it is convenient to describe them in the framework of an effective theory that arises after decoupling of the $W$-boson and all the heavier particles. The effective theory Lagrangian has the following generic form

$$
\mathscr{L}_{\text {eff }}=\mathscr{L}_{\mathrm{QCD} \times \mathrm{QED}}(\text { leptons } \& \text { quarks } \neq t)+N \sum_{n} C_{n}(\mu) Q_{n},
$$

where $Q_{n}$ are local interaction terms (operators), and $C_{n}$ are the corresponding coupling constants (Wilson coefficients) that depend on the renormalization scale $\mu$. Information on the electroweakscale physics is encoded in the values of $C_{n}$. An advantage of such a description is the possibility of resumming large logarithms $\left(\alpha_{s} \ln M_{W}^{2} / m_{b}^{2}\right)^{n}$ using renormalization group techniques, as well as an easier account for symmetries.

Most of the present article is going to be devoted to the rare decays $B_{q} \rightarrow \ell^{+} \ell^{-}$with $q=$ $s, d$ and $\ell=e, \mu, \tau$, in particular to the $B_{s} \rightarrow \mu^{+} \mu^{-}$mode which belongs to the flavour-physics highlights of the LHC. It is a strongly suppressed, loop-generated process in the SM. Its average, time-integrated branching ratio (with the final-state photon bremsstrahlung included) reads [1]

$$
\overline{\mathscr{B}}\left(B_{s} \rightarrow \mu^{+} \mu^{-}\right)_{\mathrm{SM}}=(3.65 \pm 0.23) \times 10^{-9} .
$$

The above SM prediction is based on the recent perturbative calculations of the two-loop electroweak [2] and three-loop QCD [3] corrections to the relevant Wilson coefficient. It is in agreement with the current experimental world average [4]

$$
\overline{\mathscr{B}}\left(B_{s} \rightarrow \mu^{+} \mu^{-}\right)_{\exp }=(2.9 \pm 0.7) \times 10^{-9}
$$

that has been obtained by combining the measurements of CMS [5] and LHCb [6]. These results have a significant impact on parameter spaces of various beyond-SM models. In the case of the Minimal Supersymmetric Standard Model (MSSM), they exclude a large part of the region with large $\tan \beta$ (the ratio of the two Higgs doublet vacuum expectation values). However, the moderate $\tan \beta$ region is hardly affected, especially when the superpartners are heavy enough to satisfy the Higgs mass constraints and the direct search bounds.

The operators in Eq. (1) that matter for $B_{s} \rightarrow \mu^{+} \mu^{-}$read

$$
Q_{A}=\left(\bar{b} \gamma^{\alpha} \gamma_{5} s\right)\left(\bar{\mu} \gamma_{\alpha} \gamma_{5} \mu\right), \quad Q_{S}=\left(\bar{b} \gamma_{5} s\right)(\bar{\mu} \mu), \quad Q_{P}=\left(\bar{b} \gamma_{5} s\right)\left(\bar{\mu} \gamma_{5} \mu\right) .
$$

The normalization constant in Eq. (1) in this case can be written as $N=V_{t b}^{\star} V_{t s} G_{F}^{2} M_{W}^{2} / \pi^{2}$, where $G_{F}$ is the Fermi constant (extracted from the muon decay), $M_{W}$ is the $W$-boson on-shell mass, and $V_{i j}$ are the Cabibbo-Kobayashi-Maskawa (CKM) matrix elements

In the SM, the operator $Q_{A}$ gives the dominant contribution, while the other two are negligible. Both $Q_{S}$ and $Q_{P}$ can be expressed in terms of the axial quark current

$$
Q_{S(P)} \sim\left[\bar{b} \gamma^{\alpha} \gamma_{5} s\right] \partial_{\alpha}\left[\bar{\mu}\left(\gamma_{5}\right) \mu\right]+T+E,
$$

up to total derivatives $T$ and terms that vanish by the equations of motion $E$. In effect, the $B_{s}$-meson decay constant $f_{B_{s}}$ defined by

$$
\left\langle 0\left|\bar{b} \gamma^{\alpha} \gamma_{5}\right| B_{s}\right\rangle=i p^{\alpha} f_{B_{s}}
$$


is the only non-perturbative quantity that one needs for evaluation of $\overline{\mathscr{B}}\left(B_{s} \rightarrow \mu^{+} \mu^{-}\right)$in the SM and beyond. For the numerical value of $f_{B_{s}}$, the most recent update of the $N_{f}=(2+1)$ FLAG compilation [7]

$$
f_{B_{s}}=(227.7 \pm 4.5) \mathrm{MeV}
$$

is going to be used.

Starting from Eq. (1), the following result for the average time integrated branching ratio can be derived

$$
\overline{\mathscr{B}}\left(B_{s} \rightarrow \mu^{+} \mu^{-}\right)=\frac{|N|^{2} M_{B_{s}}^{3} f_{B_{s}}^{2}}{8 \pi \Gamma_{H}^{s}} \beta\left[\left|r C_{A}-u C_{P}\right|^{2} F_{P}+\left|u \beta C_{S}\right|^{2} F_{S}\right]+\mathscr{O}\left(\alpha_{e m}\right),
$$

where $M_{B_{s}}$ is the $B_{s}$ meson mass, and $\Gamma_{H}^{s}$ stands for the total width of the heavier mass eigenstate in the $B_{s} \bar{B}_{s}$ system. The Wilson coefficients should be evaluated at the scale $\mu_{b} \sim m_{b}$. The quantities $r, \beta$ and $u$ are given by

$$
r=\frac{2 m_{\mu}}{M_{B_{s}}}, \quad \beta=\sqrt{1-r^{2}}, \quad u=\frac{M_{B_{s}}}{m_{b}+m_{s}} .
$$

In the absence of beyond-SM sources of CP-violation, we have $F_{P}=1$ and $F_{S}=1-\Delta \Gamma_{s} / \Gamma_{L}^{s}$, where $\Gamma_{L}^{s}$ is the lighter eigenstate width, and $\Delta \Gamma^{s}=\Gamma_{L}^{s}-\Gamma_{H}^{s}$. In a generic case, from the results in Ref. [8] one derives

$$
\begin{aligned}
& F_{P}=1-\frac{\Delta \Gamma^{s}}{\Gamma_{L}^{s}} \sin ^{2}\left[\frac{1}{2} \phi_{s}^{\mathrm{NP}}+\arg \left(r C_{A}-u C_{P}\right)\right], \\
& F_{S}=1-\frac{\Delta \Gamma^{s}}{\Gamma_{L}^{s}} \cos ^{2}\left[\frac{1}{2} \phi_{S}^{\mathrm{NP}}+\arg C_{S}\right]
\end{aligned}
$$

where $\phi_{s}^{\mathrm{NP}}$ describes the CP-violating "new physics" contribution to $B_{s} \bar{B}_{s}$ mixing, i.e. $\phi_{s}^{c \bar{c} s} \simeq$ $\arg \left[\left(V_{t s}^{*} V_{t b}\right)^{2}\right]+\phi_{s}^{\mathrm{NP}}$ (see Sec. 2.2 of Ref. [9]).

The results of Ref. [1] include complete corrections of order $\mathscr{O}\left(\alpha_{e m}\right)$ to the Wilson coefficient $C_{A}\left(\mu_{b}\right)$ (the only relevant one in the SM), but the $\mathscr{O}\left(\alpha_{e m}\right)$ term in Eq. (8) has been neglected. Such an approach can be justified by observing that some of the $\mathscr{O}\left(\alpha_{e m}\right)$ corrections to $C_{A}\left(\mu_{b}\right)$ get enhanced by $1 / \sin ^{2} \theta_{W}$, powers of $m_{t}^{2} / M_{W}^{2}$ or logarithms $\ln ^{2} M_{W}^{2} / \mu_{b}^{2}$, as explained in Ref. [2]. None of these enhancements is possible for the $\mathscr{O}\left(\alpha_{e m}\right)$ term in Eq. (8). This term is $\mu_{b}$-dependent and contains contributions from operators like $\left(\bar{b} \gamma_{\alpha} \gamma_{5} s\right)\left(\bar{\ell} \gamma^{\alpha} \ell\right)$ or $\left(\bar{b} \gamma_{\alpha} P_{L} c\right)\left(\bar{c} \gamma^{\alpha} P_{L} s\right)$, with photons connecting the quark and lepton lines. It depends on non-perturbative QCD in a way that is not described by $f_{B_{s}}$ alone. Its part that does depend on $f_{B_{s}}$ must compensate the $\mu_{b}$-dependence of $C_{A}\left(\mu_{b}\right)$ which amounts to about $0.3 \%$ when $\mu_{b}$ is varied from $m_{b} / 2$ to $2 m_{b}$. This is much less than the two-loop electroweak corrections to $\left|C_{A}\left(\mu_{b}\right)\right|^{2}$ that can reach a few percent level [2].

The only other possible enhancement of QED corrections might be due to soft photon bremsstrahlung. Let us consider $B_{s} \rightarrow \mu^{+} \mu^{-}(n \gamma)$ with $n=0,1,2, \ldots$. The dimuon invariant-mass spectrum in this process is obtained by summing the two distributions shown in Fig. 1. The dotted (blue) curve corresponds to the direct emission, i.e. real photon emission from the quarks. It has been estimated using Eq. (25) of Ref. [10]. The solid (red) curve is understood to describe all the other contributions to the considered process. Its tail is dominated by soft photon radiation from the muons. Interference between the two types of contributions has been neglected, as it gets 


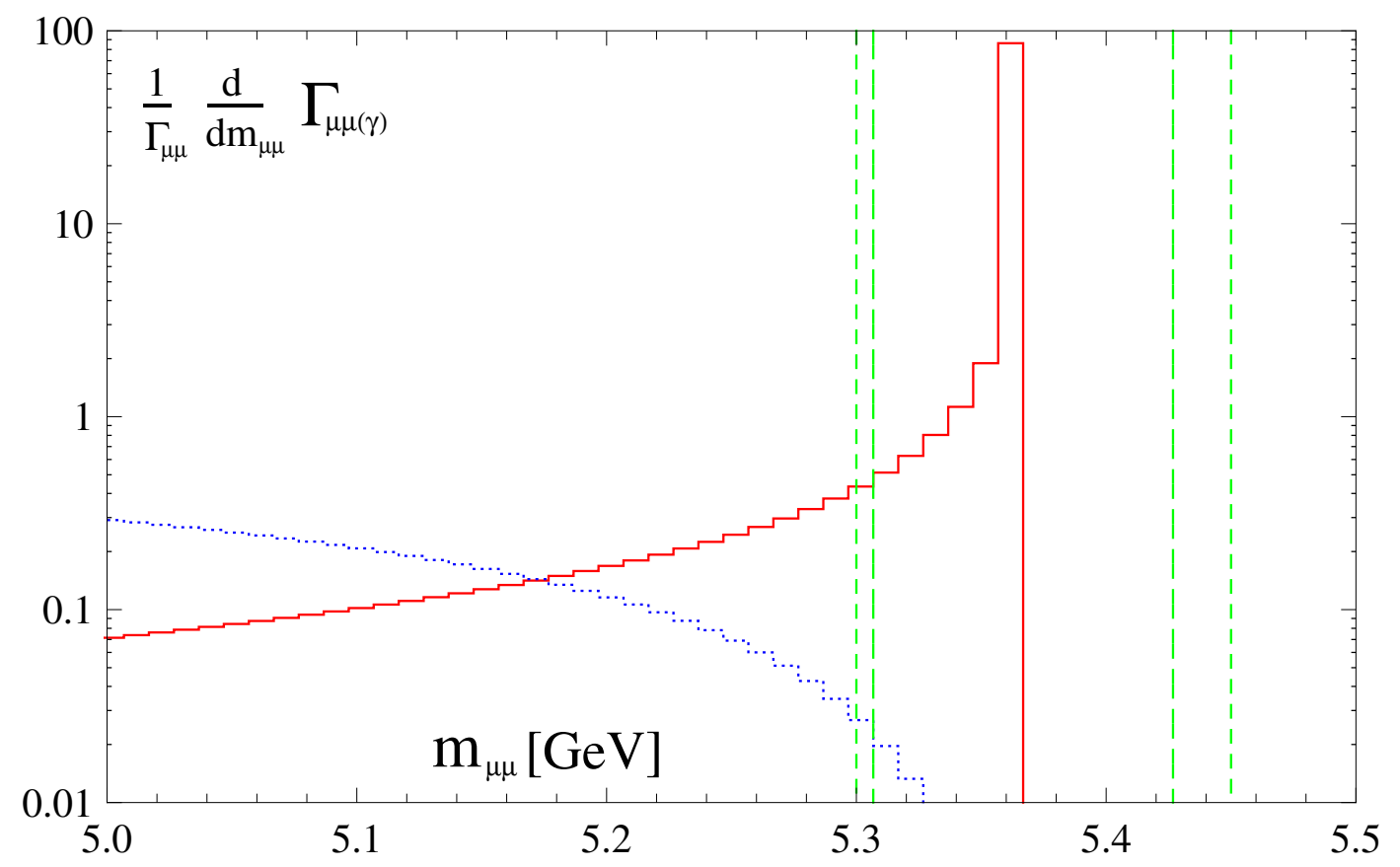

Figure 1: Contributions to the dimuon invariant-mass spectrum in $B_{s} \rightarrow \mu^{+} \mu^{-}(n \gamma)$ with $n=0,1,2, \ldots$ (see the text). Both of them are displayed in bins of $0.01 \mathrm{GeV}$ width.

suppressed by another power of $r$. The vertical dashed and dash-dotted (green) lines indicate the CMS [5] and LHCb [6] blinded signal windows, respectively. In the displayed region below the windows (i.e. between 5 and $5.3 \mathrm{GeV}$ ), each of the two types of contributions integrates to around $5 \%$ of the total rate.

The branching ratio determination on the experimental side includes a correction due to photon bremsstrahlung from the muons. For this purpose, both CMS [5] and LHCb [6] apply PHOTOS [11]. Given the current experimental uncertainties, such an approach can be understood as equivalent to extrapolating along the solid curve in Fig. 1 down to zero. In the resulting quantity, all the soft QED logarithms cancel out, and we arrive at Eq. (8), up to $\mathscr{O}\left(\alpha_{e m}\right)$ terms that undergo no extra enhancement.

The direct emission is infrared safe by itself because the decaying meson is electrically neutral. It survives in the limit $m_{\mu} \rightarrow 0$, which explains its considerable size in Fig. 1. It should be treated as background on both the experimental and theoretical sides. On the theory side, it is just excluded from $\overline{\mathscr{B}}\left(B_{s} \rightarrow \mu^{+} \mu^{-}\right)$by definition. On the experimental side, the current situation is somewhat more complex. Monte-Carlo routines are used to simulate the direct emission inside and outside the blinded windows. For the purpose of future measurements, one should either render such simulations precise (a difficult task), or restrict the actual signal windows to become as narrow as the current blinded ones, which would make the direct emission negligible. The latter solution seems to be a preferred choice, given that our knowledge of the blue curve in Fig. 1 is modeldependent and very rough.

All the input parameters that are necessary to evaluate the branching ratio in Eq. (8) are collected in Table 1 of Ref. [1]. The CKM matrix element $\left|V_{c b}\right|$ is treated in a special manner because 


\begin{tabular}{c|ccc|cc|c|c|c} 
& $f_{B_{q}}$ & $\mathrm{CKM}$ & $\Gamma_{H}^{q}$ & $M_{t}$ & $\alpha_{s}$ & $\begin{array}{c}\text { other } \\
\text { parametric }\end{array}$ & $\begin{array}{c}\text { non- } \\
\text { parametric }\end{array}$ & $\Sigma$ \\
\hline$\overline{\mathscr{B}}\left(B_{s} \rightarrow \ell^{+} \ell^{-}\right)$ & $4.0 \%$ & $4.3 \%$ & $1.3 \%$ & $1.6 \%$ & $0.1 \%$ & $<0.1 \%$ & $1.5 \%$ & $6.4 \%$ \\
$\overline{\mathscr{B}}\left(B_{d} \rightarrow \ell^{+} \ell^{-}\right)$ & $4.5 \%$ & $6.9 \%$ & $0.5 \%$ & $1.6 \%$ & $0.1 \%$ & $<0.1 \%$ & $1.5 \%$ & $8.5 \%$
\end{tabular}

Table 1: Relative uncertainties from various sources in $\overline{\mathscr{B}}\left(B_{q} \rightarrow \ell^{+} \ell^{-}\right)$. In the last column they are added in quadrature.

it is responsible for the largest parametric uncertainty. One should be aware of a long-lasting tension between its determinations from the inclusive and exclusive semileptonic decays [7]. The recent inclusive fit from Ref. [12] is adopted for our present purpose. It is the first one where both the semileptonic data and the precise quark mass determinations from flavor-conserving processes have been taken into account. Once $\left|V_{c b}\right|$ is fixed, we evaluate $\left|V_{t b}^{\star} V_{t s}\right|$ using the accurately known ratio $\left|V_{t b}^{\star} V_{t s} / V_{c b}\right|$.

Apart from the masses and couplings, the branching ratio depends on two renormalization scales $\mu_{0} \sim M_{t}$ and $\mu_{b} \sim m_{b}$ used in the calculation of the Wilson coefficient $C_{A}$. This dependence is very weak thanks to the new calculations of the two-loop electroweak and three-loop QCD corrections in Refs. [2, 3]. Here, we just fix here these scales to $\mu_{0}=160 \mathrm{GeV}$ and $\mu_{b}=5 \mathrm{GeV}$. Allowing only the top-quark mass and the strong coupling constant to deviate from their central values, one finds the following fit for the relevant Wilson coefficient in the SM: $C_{A}\left(\mu_{b}\right)=0.4690 R_{t}^{1.53} R_{\alpha}^{-0.09}$, where $R_{\alpha}=\alpha_{s}\left(M_{Z}\right) / 0.1184$ and $R_{t}=M_{t} /(173.1 \mathrm{GeV})$. The fit is accurate to better than $0.1 \%$ in $C_{A}$ for $\alpha_{s}\left(M_{Z}\right) \in[0.11,0.13]$ and $M_{t} \in[170,175] \mathrm{GeV}$. Inserting this fit into Eq. (8), and setting both $R_{t}$ and $R_{\alpha}$ to unity, one arrives at the SM result given in Eq. (2).

All the $\overline{\mathscr{B}}\left(B_{q} \rightarrow \ell^{+} \ell^{-}\right)$branching ratios calculated along the same lines yield [1]

$$
\begin{aligned}
\overline{\mathscr{B}}\left(B_{s} \rightarrow e^{+} e^{-}\right) & =(8.54 \pm 0.55) \times 10^{-14}, \quad \overline{\mathscr{B}}\left(B_{d} \rightarrow e^{+} e^{-}\right)=(2.48 \pm 0.21) \times 10^{-15}, \\
\overline{\mathscr{B}}\left(B_{s} \rightarrow \mu^{+} \mu^{-}\right) & =(3.65 \pm 0.23) \times 10^{-9}, \quad \overline{\mathscr{B}}\left(B_{d} \rightarrow \mu^{+} \mu^{-}\right)=(1.06 \pm 0.09) \times 10^{-10}, \\
\overline{\mathscr{B}}\left(B_{s} \rightarrow \tau^{+} \tau^{-}\right) & =(7.73 \pm 0.49) \times 10^{-7}, \quad \overline{\mathscr{B}}\left(B_{d} \rightarrow \tau^{+} \tau^{-}\right)=(2.22 \pm 0.19) \times 10^{-8},
\end{aligned}
$$

A summary of their error budgets is presented in Table 1. It is clear that the main parametric uncertainties come from $f_{B_{q}}$ and the CKM angles. The non-parametric uncertainty has been estimated at the level of around $1.5 \%$ of the branching ratio [1].

Let me close with briefly summarizing the current status of perturbative contributions to the $B \rightarrow X_{s} \gamma$ and $B \rightarrow X_{s} \ell^{+} \ell^{-}$branching ratios. For both these inclusive decays, non-perturbative effects come as corrections only, provided the kinematical cuts are not too stringent. Nevertheless, such non-perturbative effects are largely unknown, and remain at a few percent level. Moreover, none of the corresponding (perturbative) quark decay widths is known to $\mathscr{O}\left(\alpha_{s}^{2}\right)$ in a complete manner. In the $B \rightarrow X_{s} \gamma$ case, the main remaining issue are the interferences among the currentcurrent and the photonic dipole operators. The corresponding $\mathscr{O}\left(\alpha_{s}^{2}\right)$ terms were calculated in the heavy charm limit $\left(m_{c} \gg m_{b} / 2\right)$ in Ref. [13], while a calculation in the $m_{c}=0$ case is being finalized [14]. Once completed, the $m_{c}$-interpolation of Ref. [15] and the phenomenological results of Ref. [16] are going to be updated. 
In the $B \rightarrow X_{s} \ell^{+} \ell^{-}$case, there is only one Wilson coefficient that is not yet known up to $\mathscr{O}\left(\alpha_{s}^{2}\right)$, namely the one of the operator $Q_{9} \sim\left(\bar{b}_{L} \gamma^{\alpha} s_{L}\right)\left(\bar{\ell} \gamma_{\alpha} \ell\right)$. Such a calculation is technically feasible, following the same methods as, e.g., in Ref. [3]. However, it remains an open question whether a phenomenological motivation for this task is already sufficient at present.

To conclude, the rare leptonic, semileptonic and radiative $B$-meson decay modes provide important constraints on beyond-SM physics, and require precise perturbative calculations within the SM. Recently calculated corrections to the Wilson coefficients significantly improve the accuracy in the $B_{q} \rightarrow \ell^{+} \ell^{-}$case, and are relevant for $B \rightarrow X_{s} \ell^{+} \ell^{-}$, too. As far as $B \rightarrow X_{s} \gamma$ is concerned, all the relevant Wilson coefficients are already known up to $\mathscr{O}\left(\alpha_{s}^{2}\right)$, and the main remaining task is completing the evaluation of the on-shell matrix elements to this order. All these calculations are necessary to match the accuracy of the present and planned experimental determinations of the corresponding branching ratios.

\section{References}

[1] C. Bobeth, M. Gorbahn, T. Hermann, M. Misiak, E. Stamou and M. Steinhauser, Phys. Rev. Lett. 112 (2014) 101801 [arXiv:1311.0903].

[2] C. Bobeth, M. Gorbahn and E. Stamou, Phys. Rev. D 89, 034023 (2014) [arXiv:1311.1348].

[3] T. Hermann, M. Misiak and M. Steinhauser, JHEP 1312, 097 (2013) [arXiv:1311.1347].

[4] CMS and LHCb Collaborations, Conference Report No. CMS-PAS-BPH-13-007, LHCb-CONF-2013-012, http: / / cds . cern. ch/record/1564324.

[5] S. Chatrchyan et al. (CMS Collaboration), Phys. Rev. Lett. 111, 101804 (2013) [arXiv:1307.5025].

[6] R. Aaij et al. (LHCb Collaboration), Phys. Rev. Lett. 111 101805, (2013) [arXiv:1307.5024].

[7] S. Aoki et al. (Flavour Lattice Averaging Group), arXiv:1310.8555; updates at http://itpwiki.unibe.ch/ flag.

[8] K. De Bruyn et al., Phys. Rev. Lett. 109, 041801 (2012) [arXiv:1204.1737].

[9] A. J. Buras, R. Fleischer, J. Girrbach and R. Knegjens, JHEP 1307 (2013) 77 [arXiv:1303.3820].

[10] Y. G. Aditya, K. J. Healey and A. A. Petrov, Phys. Rev. D 87, 074028 (2013) [arXiv:1212.4166].

[11] P. Golonka and Z. Was, Eur. Phys. J. C 45, 97 (2006) [hep-ph/0506026].

[12] P. Gambino and C. Schwanda, Phys. Rev. D 89, 014022 (2014) [arXiv:1307.4551].

[13] M. Misiak and M. Steinhauser, Nucl. Phys. B 840, 271 (2010) [ arXiv:1005.1173].

[14] M. Czakon, P. Fiedler, T. Huber, M. Misiak, T. Schutzmeier and M. Steinhauser, in preparation.

[15] M. Misiak and M. Steinhauser, Nucl. Phys. B 764, 62 (2007) [hep-ph/0609241].

[16] M. Misiak et al., Phys. Rev. Lett. 98, 022002 (2007) [hep-ph/0609232]. 